\title{
Subtypisierung soll den therapeutischen Durchbruch bringen
}

Bisher sind die therapeutischen Erfolge beim Sjögren-Syndrom begrenzt. Die Hoffnungen richten sich nun auf eine Subtypisierung anhand derer zielgerichtete Therapien eingesetzt werden sollen.

Obwohl das Sjögren-Syndrom schon lange bekannt ist und die häufigste Kollagenose darstellt, sind die therapeutischen Erfolge bisher begrenzt. Eine Wirkung auf die SiccaSymptomatik ist für Pilocarpin sowie Cortison- und Ciclosporin-Augentropfen durch randomisierte, kontrollierte Studien gesichert, wie Prof. Dr. Torsten Witte, Klinik für Immunologie und Rheumatologie, Medizinische Hochschule Hannover, in der Sitzung „WIN - Therapiefortschritte bei Kollagenosen" berichtete.

\) Nach neuen Untersuchungen sind anhand der Klinik vier Cluster des Sjögren-Syndroms zu unterscheiden

Ein leichter positiver Effekt auf die Speichelproduktion sei für orales Interferon alpha und Ciclosporin p.o. belegt. Bei neueren Immunsuppressiva, wie Methotrexat, Leflunomid, Mycophenolat-Mofetil und Azathioprin waren die Ergebnisse dagegen bisher enttäuschend. Grund hierfür scheint zu sein, dass nach neueren Untersuchungen anhand der Klinik vier Cluster des Sjögren-Syndroms zu unterscheiden sind:

Quelle: SpringerMedizin.de, Dr. Wiebke Kathmann, basierend auf dem 47. Kongress der Deutschen Gesellschaft für Rheumatologie (DGRh), 4.-7. September 2019, Dresden
Lungenerkrankung (ILD), Polyneuropathie, Serositis und Nephritis (6\%) und

- Subgruppe 4 zusätzlich zur SiccaSymptomatik eine Purpura und Polyneuropathie (10\%).

Basierend auf dem großen europäischen Projekt PRICISESADS, das eine Subtypisierung anhand von Genom, Serologie, Metabolom und Transkriptom erstellt, ist sogar von mindestens fünf Subtypen auszugehen, sagte Witte. Dies könne erklären, warum die Studien zu modernen Therapien bisher negativ ausgefallen seien, da sie oft nur ein Cluster auf's Korn nähmen, während die Studienpopulation gemischt sei. Die Medikamente könnten sich also als effizienter erweisen, wenn die Patienten besser klassifiziert seien.

\section{Erste erfolgreiche Kombinations- therapie}

Bis es so weit ist, ruht die Hoffnung auf Kombinationstherapien. Denn so können mehrere pathophysiologisch relevante Zelltypen gleichzeitig ins Visier genommen werden. Für die Kombination aus Leflunomid und Hydroxychlorochin konnte der Wirksamkeitsnachweis in einer beim Kongress des American College of Rheumatology (ACR) 2018 vorgestellten placebokontrollierten, doppelblinden, randomisierten Studie erbracht werden [1].

Der Endpunkt für extraglanduläre Manifestationen ESSDAI (EULAR
Sjögren's syndrome disease activity index) sowie der ESSPRI (EULAR Sjögren's syndrome patient reported index) hatten sich innerhalb von 24 Wochen signifikant gebessert. Zudem hatte der Speichelfluss deutlich zugenommen.

》) Medikamente könnten sich als effizienter erweisen, wenn die Patienten besser klassifiziert sind

Hoffnungsträger bei den Monotherapien sind derzeit Abatacept, für das ein dramatischer Effekt auf den ESSDAI gezeigt werden konnte [2] und lanalumab (ein BAFF-Rezeptor-Blocker), das nach Einmalgabe zu einer potenten und anhaltenden B-ZellDepletion führte, die eine Verbesserung mit sich brachte [3].

\section{Literatur \\ 1. Radstake T et al (2019) ACR 2019, Abstract L10; van der Heijden EHM et al. Expert Review of Clin Immunol. 15(7):801-808 \\ 2. Baer A et al (2019) EULAR, OP0039 \\ 3. Dörner T et al (2019) Ann Rheum Dis. 78:641-647}

rheuma plus $2019 \cdot 18: 212$ https://doi.org/10.1007/s12688-01900305-6

(c) Springer-Verlag GmbH Austria, ein Teil von Springer Nature 2019 